Research Article

\title{
Simulation and Experiment of New Ultrasonic Vibration Network
}

\author{
Xiwen Chen $\left(D,{ }^{1}\right.$ Xiaoqian $\mathrm{Li}^{1}{ }^{1}$ and Weihua Gui ${ }^{2}$ \\ ${ }^{1}$ Light Alloy Research Institute, Central South University, Changsha 410083, China \\ ${ }^{2}$ School of Information Science and Engineering, Central South University, Changsha 410083, China \\ Correspondence should be addressed to Xiwen Chen; chenxiwen001@qq.com
}

Received 22 July 2019; Revised 15 November 2019; Accepted 21 November 2019; Published 16 October 2020

Academic Editor: Luca Landi

Copyright (c) 2020 Xiwen Chen et al. This is an open access article distributed under the Creative Commons Attribution License, which permits unrestricted use, distribution, and reproduction in any medium, provided the original work is properly cited.

\begin{abstract}
A reasonable ultrasonic vibration network can improve the casting quality of aluminum alloy. Ultrasonic vibration network based on a honeycomb structure has been designed, referred to as a new vibration network. The new vibration network can solve the problems of nonuniform distribution of power ultrasonic wave, small working area and low volume of ultrasonic vibration network, low efficiency of the frequency spectrum and power spectrum, and poor quality of aluminum alloy casting. The number of vibration nodes can be determined based on the number of layers of the vibration source nodes. The edge length of regular hexagonal honeycomb cells can be determined based on the size of the casting ingot. The output power and resonant frequency of the ultrasonic vibration network can be adjusted in real time according to the status of aluminum alloy melt. A seven-node new ultrasonic network and a four-node ultrasonic network with a traditional structure were selected and used in the experiment and simulation of a $500 \mathrm{~mm}$ diameter 2219 aluminum alloy ingot. In comparison with the traditional four-node ultrasonic network, the effective volume and area, frequency spectrum efficiency, and comprehensive coverage probability of the seven-node new ultrasonic vibration network increased by $34.06 \%, 23.12 \%, 17.25 \%$, and 0.308 , respectively. The difference between the desired value and average efficiency of the power spectrum was $0.292 \mathrm{~W} / \mathrm{cm}^{2}$, and the average grain size of aluminum alloy decreased by 34.98 microns. These results indicate that the efficiency of ultrasonic-vibration-assisted casting system and the quality of aluminum alloy casting can be improved using the new ultrasonic vibration network.
\end{abstract}

\section{Introduction}

A suitable structure of an ultrasonic vibration network is beneficial for improving the spatial efficiency of an ultrasonic-vibration-assisted casting system. A reasonable structure of an ultrasonic vibration network ensures a uniform grain size of aluminum alloy melt, considerably reduces the probability of void formation, and stabilizes the output power and harmonic frequency. Therefore, building a scientific ultrasonic vibration network and improving the efficiency of ultrasonic-vibration-assisted casting system has attracted a considerable amount of research focus in the field of aluminum alloy casting [1-5]. In the traditional ultrasonic-vibration-assisted casting process, the tool heads of the ultrasonic vibration network are not dependent on each other. The four-node ultrasonic vibration network is basically used in the ultrasonic-vibration-assisted casting process of aluminum alloy ingots with diameters less than
$1300 \mathrm{~mm}$; in such a network, the number of horns is constant. Ultrasonic power refers to the power consumed by the ultrasonic vibration power supply to generate ultrasonic field effect on the ultrasonic vibration network, which results in an uneven distribution ultrasonic power of the ultrasonic in ultrasonic-vibration-assisted casting process, and the effective area and volume are limited [5-8]. In the traditional ultrasonic-vibration-assisted casting process of 2219 aluminum alloy ingot with a diameter of $630 \mathrm{~mm}$, the four-node ultrasonic vibration network is adopted. The effective area ratio of the ultrasonic effect is $40.6 \%$, and the effective volume ratio of the ultrasonic effect is $30.23 \%$. Furthermore, the efficiency of traditional ultrasonic vibration network space is low, and the efficiency of the ultrasonic-vibrationassisted casting process is unsatisfactory.

The output power and harmonic frequency of the traditional ultrasonic vibration network are not adjusted in real time in accordance with the situation. The output power provided 
by ultrasonic vibration power supply is unstable, the frequency is out of tune, the ultrasonic-vibration-assisted casting system is unstable, and the quality of the cast aluminum alloy is low.

It is important to design a highly efficient and reliable ultrasonic vibration network for improving the efficiency of an ultrasonic-vibration-assisted casting system [9-12]. A new ultrasonic vibration network has been designed based on the working principle of the communication network with honeycomb structures. The network can determine the number of vibration nodes based on the diameter of an aluminum alloy ingot.

The efficiency of the casting network is considerably improved using a hexagonal symmetry mechanism. The output power and harmonic frequency of the ultrasonic vibration network are adjusted in real time depending on the actual situation. The output power and frequency are stable, the high temperature and high pressure caused by power ultrasonic wave are constant [13-16], the grain size of the aluminum alloy is uniform [17-19], and the working efficiency of the ultrasonic-vibration-assisted casting system and the casting quality are improved.

\section{New Ultrasonic Vibration Network Design}

2.1. Vibration Network. A communication network based on a honeycomb structure includes six boundary nodes and one central node. An equilateral triangle is formed by every three adjacent nodes, the coverage area occupied by each node is equal, the difference between signal intensity distribution and attenuation is reduced, and the quality of communication is improved. The new ultrasonic vibration network determines the number of vibration nodes based on the number of layers and scale of the ultrasonic vibration network. The number of vibration elements in an ultrasonic vibration network with $L$ layers can be determined using the following formula:

$$
N_{\mathrm{vnod}}=2\left(L^{2}+\sum_{i=1}^{L-2} i\right)-1
$$

The number of layers from the middle to the edge of the hexagon is $L$, and the new ultrasonic vibration network system with 1,7 , and 37 heads connecting the central points of each hexagon contains the single layer, two layers, and 4 layers, respectively. The structure is shown in Figure 1.

When the connection between the nodes of an ultrasonic vibration network is strong, the frequency of adjacent nodes is not synchronized, which will lead to resonance, cancellation, and detuning of power of ultrasonic, and the efficiency of the horns will be reduced. To some extent, the use of an "amplifier is not connected of physically but connected on ultrasonic vibration field," the new ultrasonic vibration network structure can reduce interference between rods, ensure that the horns work independently and stably, and improve work efficiency.

Coverage probability refers to the network coverage mechanism of a mobile communication network based on a honeycomb structure to improve the effective space occupied by an ultrasonic vibration network. The honeycomb structure of the ultrasonic vibration network in two-dimensional plane and its distribution density is defined as $\lambda_{B}$. Any point in the

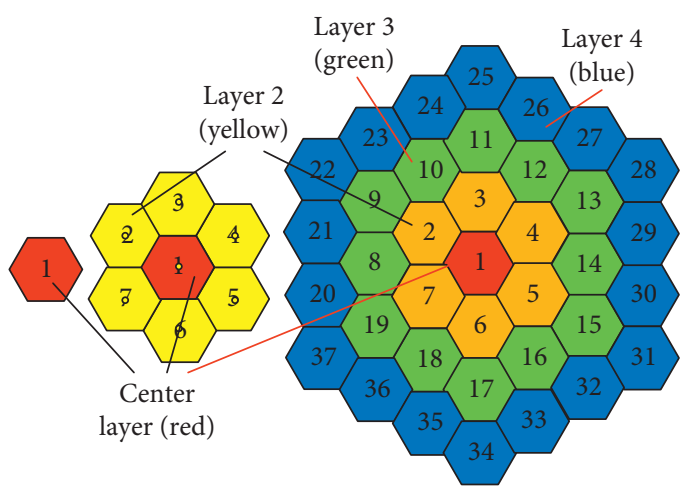

FIGURE 1: Ultrasound vibration network based on honeycomb structure.

vibration network will be affected by three horns in the new ultrasonic vibration network. The distance between the detection point and rod $i$ is defined by its coordinate $R(i)$, square distribution of $i$ is $\Gamma(i)$, so the distribution of the vibration network coverage is given by formulas (2) and (3)

$$
\begin{gathered}
P_{c}^{A} \approx P\left(\bigcap_{i=1}^{N_{\text {vod }}} A_{i}\right), \\
R(i)=e^{-\lambda_{B} \pi r^{2}} \frac{2(\lambda B \pi r)}{\Gamma(i)}\left(1-10^{-L N \mathrm{vod}}\right) .
\end{gathered}
$$

In the above formulas, $i=1,2,3, \ldots, 7 ; r=10.5 \mathrm{~cm}$; and $A_{i}$ is the covering area of horn $i$. Each honeycomb unit consists of six boundary points and one center points, the vibration network of the seven rods has 49 points for get the information of vibration network, and the distribution density is 0.01573 points per centimeter. In the formula, $\xi$ is the area of a circle with radius of $R(i), \varepsilon$ is the characteristics of a repeat tail with distance from boundary to the center points on ultrasonic horn icovered, and the range of $\varepsilon$ is 1 to 3 .

$$
P\left(A_{i}\right)=1-\int_{0}^{105} \int_{106}^{182} \frac{\varepsilon D^{(-\varepsilon-1)} \cdot e^{-\lambda_{B} \pi(D+\zeta)^{i}}}{(R(i)+\varsigma)\left(106^{-\varepsilon}-182^{-\varepsilon}\right) \Gamma(i)} \mathrm{d} D \mathrm{~d} \varsigma .
$$

\subsection{Spatial Efficiency}

\subsubsection{Spatial Efficiency of the Traditional Vibration Network.} In the ultrasonic-vibration-assisted investment casting of aluminum alloy ingots with different specifications, the bottom of the tool head is vertically downward. The area where the temperature of the sampling points is higher than the average melt temperature in the molds is defined as the ideal action zone of the honeycomb unit. The average temperature of the sampling points within $0.1-5.689 \mathrm{~cm}$ vertically downward from the bottom of the traditional ultrasonic vibration network tool head is $15.69^{\circ} \mathrm{C}$ higher than that of the melt. The average temperature of the sampling points in the aluminum alloy melt within 
$0.12-4.321 \mathrm{~cm}$ vertically downward from the bottom of the honeycomb structure ultrasonic network tool head is $15.212^{\circ} \mathrm{C}$ higher than the average temperature of the aluminum alloy melt, and the distance from the downstairs surface of head to ultrasonic efficiency surface is $5.01 \mathrm{~cm}$, which is the unified ideal depth of action for different structures of ultrasonic networks.

The diameter of the traditional ultrasonic vibration network tool head is $10 \mathrm{~cm}$, and the functional analysis of a single tool head is shown in Figure 2. Based on the previous analysis, the ideal area and volume of the ultrasonic vibration network consisting of $n$ horns are as follows.

$$
\begin{gathered}
S_{\text {nor }}=4 n \pi r_{\text {ult }}^{2}+10^{-2 n} r, \\
V_{\text {nor }}=3 n \pi r_{\text {ult }}^{2} h+10^{-3 n} r .
\end{gathered}
$$

Here, $r_{\text {ult }}$ is the radius of the tool head and $h$ is the distance from the bottom of the tool head to the working surface. The radio of the tool head in the traditional sevenbar ultrasonic vibration network is $5 \mathrm{~cm}$. The cross-sectional area and volume of formulas (5) and (6) are $1961.1 \mathrm{~cm}^{2}$ and $9820.5 \mathrm{~cm}^{3}$, respectively. The cross-sectional area of the 2219 aluminum alloy ingot is $3115.665 \mathrm{~cm}^{2}$; in this study, the optimal depth at which the ultrasonic-vibration-assisted casting system is effective is $5 \mathrm{~cm}$. The optimal volume of action is $15578.325 \mathrm{~cm}^{3}$, and the volume efficiency of casting increases from $43.54 \%$ to $63.039 \%$.

\subsubsection{Spatial Efficiency Analysis of the Honeycomb Network.} The new ultrasonic vibration network was constructed for a 2219 aluminum alloy ingot by using seven ultrasonic horns which is shown in Figure 3.

The side length of the honeycomb hexagonal unit is $a$, the area is $S_{\mathrm{HCL}}$, the number of layers of the vibration network is $L$, and the diameter of the ingot is $d_{\mathrm{ci}}$. Thus, the cross-sectional area and volume of the ultrasonic vibration network with a honeycomb structure with $V_{\mathrm{HCL}}$ of $L$ layers are given by formulas (7) and (8):

$$
\begin{aligned}
S_{\mathrm{HCL}} & =\frac{2 \sqrt{3}\left(d_{\mathrm{ci}}\right)^{2}}{(4 L-1)^{2}} \times N_{\mathrm{vnod}}+\frac{\pi\left(d_{\mathrm{ci}}\right)^{2}}{\left(2 N_{\mathrm{vnod}}\right)^{2 L}}, \\
V_{\mathrm{HCL}} & =\frac{10 \sqrt{3}\left(d_{\mathrm{ci}}\right)^{2}}{(4 L-1)^{2}} \times N_{\mathrm{vnod}}+\frac{\pi\left(d_{\mathrm{ci}}\right)^{3}}{\left(4 N_{\mathrm{vnod}}\right)^{3 L}} .
\end{aligned}
$$

The number of cellular cells in the ultrasonic vibration network is closely related to the size of the ingot and the number of network layers. The hexagonal cylinders at the front of the tool head can be replaced depending on the size of the ingot and the number of network layers. The unit side length (a) for the two-layer honeycomb structure of the ingot with a diameter of $630 \mathrm{~mm}$, the side length of hexagon with ultrasonic horn, is $103.9 \mathrm{~mm}$, and the three views of the ultrasonic vibration network header of the horn based on the honeycomb structure are shown in Figure 4.

The ideal working depth of the ultrasonic vibration network head of horn with honeycomb structure adopts a

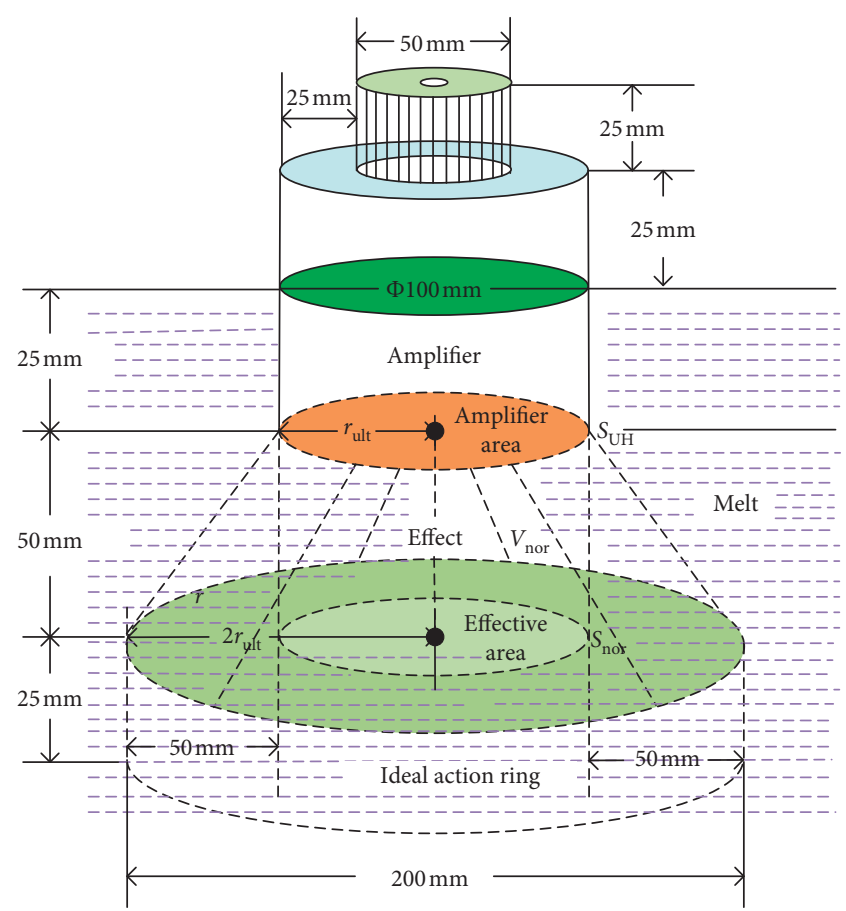

FIGURE 2: Diagram of traditional ultrasonic vibration network horn action.

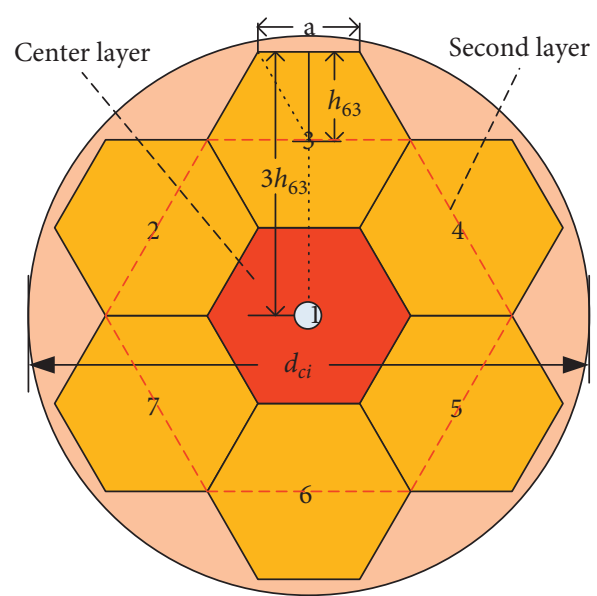

FIgURE 3: Two-layer hypernetwork of new acoustic vibration.

unified ideal acting depth. The cross-sectional area, acting volume, and efficiency of the ultrasonic vibration network of the honeycomb structure with a depth of $5 \mathrm{~cm}$ and consisting of 2 to 6 layers are listed in Table 1 .

As the number of honeycomb cell layers increases, the crosssectional area and volume of the vibration network continue to increase. Additionally, the working efficiency gradually increases, but the rate of increase rate gradually decreases.

\subsection{Power Load and Resonant Frequency Output}

2.3.1. Power Load. The average acting force and instantaneous response speed of horn $i$ are $F_{\text {iave }}$ and $V_{\text {iave }}$, respectively, $T=2 \pi / f$ is the cycle of vibration, and $a=1.001$. 


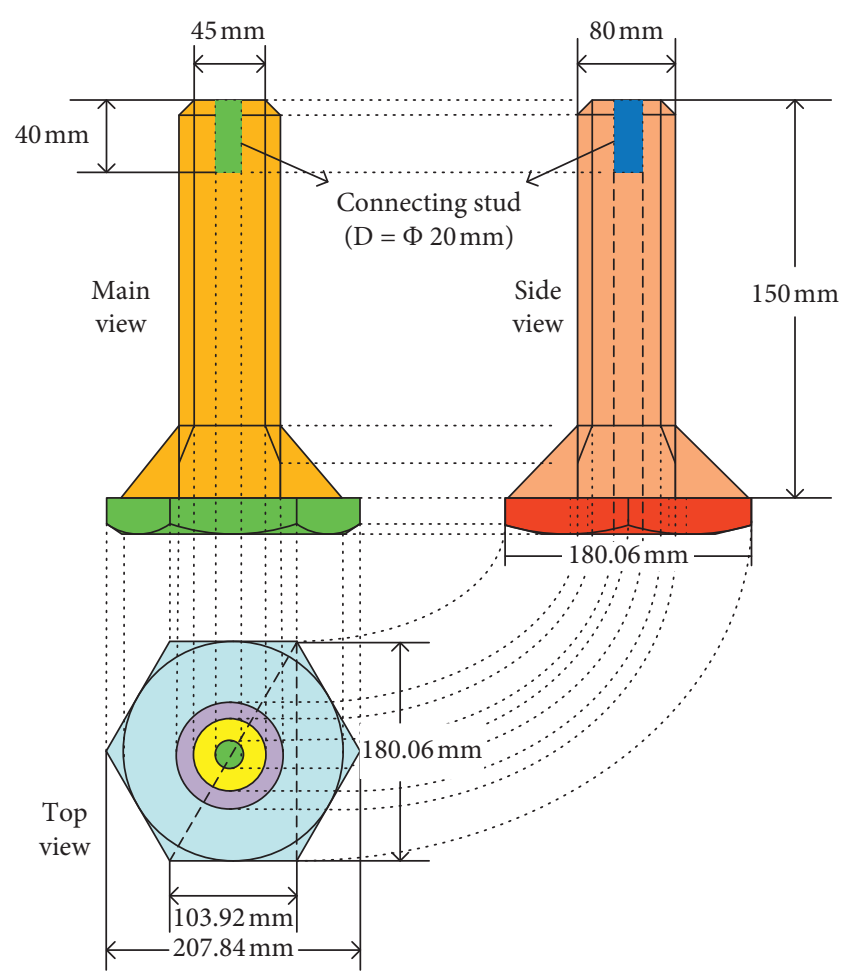

Figure 4: Construction of ultrasonic vibration network tool head for honeycomb structure.

TABLE 1: Efficiency of ultrasonic vibration network honeycomb structure with different layers.

\begin{tabular}{lcccc}
\hline Layers & Nodes & Area $\left(\mathrm{cm}^{2}\right)$ & Volume $\left(\mathrm{cm}^{3}\right)$ & Efficiency $(\%)$ \\
\hline 2 & 7 & 1964.1 & 9820.52 & 63.05 \\
3 & 19 & 2158.9 & 10794.43 & 69.32 \\
4 & 37 & 2260.9 & 11304.41 & 72.63 \\
5 & 61 & 2323.2 & 11615.94 & 74.61 \\
6 & 91 & 2365.1 & 11825.45 & 75.95 \\
7 & 127 & 2395.2 & 11975.81 & 76.96 \\
8 & 169 & 2417.8 & 12089.14 & 77.68 \\
\hline
\end{tabular}

$$
\begin{aligned}
P_{\text {iaf }}(t) & =\frac{a \int_{0}^{T} F_{\text {iave }} \times V_{\text {iave }} \cos (f t) \cos (f t+\phi) \mathrm{d} t}{T}, \\
V_{\text {iave }} & =2 a \int_{0}^{360} a_{i}(t) f_{i}(t) \mathrm{d} t+\frac{1}{360 N_{\text {vnod }}}, \\
F_{\text {iave }} & =2 a m_{i} \int_{0}^{360}\left(f_{i}(t)-f_{i}(t-1)\right) \mathrm{d} t .
\end{aligned}
$$

Here, $\mathrm{A}$ is the amplitude of the horn $i$ on $t, f_{i}(t)$ and $f_{i}(t-1)$ are the frequencies of the horniat $t$ and $t-1$ moment, respectively; $m_{i}$ is the mass of the horn $i$; and $\phi$ is the phase shift. Forecasting the normal component load is important for forecasting the power load of ultrasonic vibration power supply [17-20]. Thus, the load forecasting model is given by formulas (11) and (12).

$$
\begin{aligned}
p_{g}(t) & =\prod_{h=590}^{765} p_{s} \sigma_{\text {tem_al }}(t)+\frac{1}{360} \sum_{t=1}^{360}\left(\int_{0}^{6} u_{i}(t) d i\right) \\
\sigma_{\text {tem_al }}(t) & =\frac{T_{\mathrm{al}}(t)-T_{\mathrm{al}}(t-1)}{735}+\frac{1}{735 N_{\mathrm{vnod}}}
\end{aligned}
$$

In these formulas, $p_{g}(t)$ is the normal load forecasting in 300 seconds, $u_{i}(t)$ is the voltage at time $t$ of horn $i, p_{s}$ is the rated power of ultrasonic power supply, $\sigma_{\text {tem_al }}(t)$ is the temperature influence factor of the melt of the new ultrasonic vibration network at moment $t$, and $T_{\mathrm{al}}(t)$ is the temperature melt on $t$. Referring to Box-Jenkins method, the forecasting model based on the historical power load is designed as follows:

$$
P_{\text {hisi }}(t)=p_{a}(t-2)-\sum_{t=1}^{t-1} \frac{a(t) R_{i}(t) I^{2}(t)}{t}
$$

where $P_{\text {hisi }}(t)$ represents the forecasting value based on historical power load, $p_{a}(t-1)$ is the average load before time $t, R_{i}(t)$ is the internal resistance of power supply at time $t, I(t)$ is the current at time $t, G$ is the load correction factor before time $t, I$ is $0.89-1.09$, and $P_{\text {avn }}(t)$ is the load of the $\mathrm{N}$-BAR honeycomb ultrasonic vibration network and obtained by setting the proportion of the first four aspects to be $25 \%$.

$$
\begin{aligned}
P_{\mathrm{avn}}(t) & =\frac{\sum_{i=1}^{n}\left[P_{i \mathrm{af}}(t)+P_{g i}(t)+P_{\mathrm{hisi}}(t)+P_{i}(t)\right]}{4}+\delta_{n}(t), \\
\delta_{n}(t) & =P_{\mathrm{avn}}(t)-P_{\mathrm{avn}}(t-1) .
\end{aligned}
$$

In the formula, $n$ denotes the number of horn nodes, and the power supply of the 2219 aluminum alloy ultrasonicvibration-assisted casting system for $630 \mathrm{~mm}$ is set at $20 \mathrm{kHz}$. The power supply is sampled at intervals of $1 \mathrm{~s}$, and the time slice is $3 \mathrm{~min}$. The output power of the horn $i$ is obtained as follows:

$$
\begin{aligned}
& P_{i \mathrm{o}}(t)=r_{i p}(t)+\frac{2\left(p_{s}+p_{i \mathrm{in}}(t) \eta\right)\left(\int_{0}^{2 \pi} v_{i s}+\sum_{t=1}^{180} a_{i t} \cos \left(i \omega_{i}(t)\right) \mathrm{d}\left(\omega_{i}\right)\right)}{U_{\text {in }}(t) \alpha \eta \pi}, \\
& r_{i p}(t)=\frac{\sum_{t=1}^{180}\left(p_{\text {imax }}(t)+p_{\text {iave }}(t)\right)}{180 k},
\end{aligned}
$$

where $p_{i \text { in }}(t)$ and $p_{\text {io }}(t)$ are input and output powers of horn $i$ at time $t, p_{i \max }(t)$ and $p_{\text {iave }}(t)$ are the maximum power and average output power before time $t, \omega$ is the angular frequency, $v_{i s}$ is the input voltage of horn $i$, and $\eta$ and $\alpha$ are inverting efficiency and conductivity, respectively.

2.3.2. Resonant Frequency. The analysis of the resonant frequency of the horn in the ultrasonic vibration network is performed using the basic input data of the ultrasonic vibration power supply and resonant frequency of historical and resonant frequency of forecasting. The resonant 
frequency of the horn in the vibration network at $180 \mathrm{~s}$ is as follows:

$$
\begin{aligned}
& f_{i 0}(t)=r_{i f}(t-1)+\frac{\sum_{t=1}^{360}\left(f_{\text {iave }}(t-1)+r_{i f}(t-1)\right)}{360}, \\
& r_{i f}(t)=\frac{1}{360} \sum_{t=1}^{360} f_{i h}(t)+\frac{1}{2 t} \sum_{i=1}^{t} r_{i f \text { ave }}(t-1),
\end{aligned}
$$

where $f_{i 0}(t)$ is the average output frequency in the first terms of $0.5 \mathrm{t}$ of horn $i, r_{i f}(t-1)$ is the error of frequency in terms of $0.5(t-1)$ with horn $i, f_{\text {iave }}(t-1)$ is the average output $t$ frequency in terms of $0.5(t-1)$ with horn $i, f_{i h}(t)$ is the highest frequency in terms of $0.5 t$ with horn $i$, and $r_{i f a v e}(t-1)$ is the average of frequency error in terms $0.5(t-1)$ with horn $i$. Thus, the frequency with horn $i$ at the time of $0.5 t$ is given by

$$
f_{i}(t)=a_{i}(t) \sin \left(\frac{2 f_{i}\left(t L_{d}+h \cos \alpha\right)}{c}\right)+r_{i f}(t) .
$$

In the formula, the propagation speed of ultrasound is $c$, and $L_{d}$ is the distance from the center to the measured point of horn $i$. The resonant frequency of the ultrasonic network of horn $i$ at time $t$ is deduced by Hilbert transform. $C$ represents the frequency of horn $i$ at time $t$.

\section{Simulation and Analysis}

3.1. Coverage Analysis. Considering the influence of the loss of isotropic and anisotropic paths on the coverage probability of the ultrasonic vibration network, the simulation parameters are listed in Table 2.

When the average loss index of the path is A threshold, $T_{\text {sir }} \leq 14 \mathrm{~dB}$, the loss of the isotropic path is ignored, and the coverage probability of the loss of the anisotropic path decreases.

When $T_{\text {sir }} \geq 15 \mathrm{~dB}$, the coverage probability of the loss of the isotropic path decreases, and the coverage probability of the loss model of the anisotropic path increases.

The average comprehensive coverage probability of the test point is 0.82 , which is 0.33 higher than that of the traditional ultrasonic network. Figure 5 shows the simulation curve.

3.2. Power Spectrum Density Simulation Analysis. The new vibration network uses seven vibration sources in assisted casting process, and the area of action of each rod is $S_{\mathrm{Hu}}=2 \sqrt{3}\left(d_{\mathrm{ci}}\right)^{2} /(4 L-1)^{2}$. The traditional ultrasonic vibration network uses four vibration sources, and the area of action of each rod is $S_{\mathrm{Gu}}=4 \pi\left(r_{\mathrm{ult}}\right)^{2}$, and the power spectral efficiency analysis model of the new ultrasonic vibration network and the traditional structure is established.

$$
\begin{aligned}
P_{\mathrm{Hc}}(j) & =\int_{0}^{\pi / 2} \frac{P_{j}(t)(\sin (2 \pi \beta)+\cos (2 \pi \beta))}{S_{\mathrm{Hu}}} \mathrm{d} \beta, \\
P_{\mathrm{Gc}}(i) & =\int_{0}^{\pi / 2} \frac{d_{i}(t) p_{i}(t) \cos \alpha}{d_{i}(t-1) S_{\mathrm{Gu}}} \mathrm{d} \alpha .
\end{aligned}
$$

TABLE 2: Simulation parameters of ultrasonic vibration network.

\begin{tabular}{lc}
\hline Subject & Settings \\
\hline Power of horn $i$ & $p_{i}=250 \mathrm{w}$ \\
Amplifier number & $N_{u}=7$ \\
Test number & $N_{\text {Tnode }}=2$ \\
Maximum value of amplifier $i$ coverage & $\psi_{i}=15.0 \mathrm{~cm}$ \\
Minimum value of amplifier $i$ coverage & $v_{i}=5.0 \mathrm{~cm}$ \\
Loss size of amplifier & $\theta_{i}=1.0 \mathrm{~mm}$ \\
Frequency multiplexing factor & $\delta=3$ \\
\hline
\end{tabular}

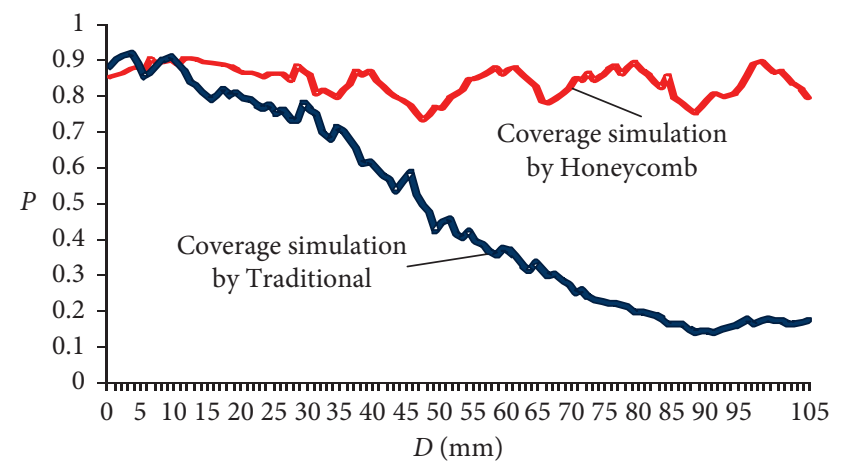

Figure 5: Coverage comparison of simulation in new and traditional ultrasonic vibration networks.

In the simulation of power spectrum efficiency with 2219 aluminum alloy assistant casting process, the total rated power of each vibration source of a seven-bar honeycomb structure and a four-bar traditional structure vibration network is $250 \mathrm{~W}$. The power spectrum efficiency curve simulated using MATLAB is shown in Figure 6.

The power density of the new vibration network is $0.736-0.906 \mathrm{~W} / \mathrm{cm}^{2}$, which is closer to the ideal value of $0.556 \mathrm{~W} / \mathrm{cm}^{2}$. It has an area ratio of $63.04 \%$. The work distribution is uniform, the area of action is large, and the work efficiency and reliability are improved. The unit load range of the traditional structural vibration network is $0.07-3.01 \mathrm{~W} / \mathrm{cm}^{2}$, and the effective area ratio is only $20.52 \%$.

3.3. Simulation of Frequency Tracking. Frequency tracking simulation analysis is similar to load simulation analysis. The conditions of detuning and resonance of ultrasonic vibration network are judged according to the resonant frequency of the honeycomb unit. The efficiency of the honeycomb-like ultrasonic vibration network is higher than that of the conventional ultrasonic vibration network. The resonance frequency curve of the ultrasonic vibration network simulated using MATLAB is shown in Figure 7.

The results show that the power load and harmonic frequencies of the horns follow the same trend. The frequency range is $18.01 \mathrm{kHz}-19.82 \mathrm{kHz}$. The error between the simulation results and the ideal values is within a controllable range. The frequency range of the traditional vibration network is $18.74-20.01 \mathrm{kHz}$. The frequency range of the honeycomb network is larger than that of the traditional ultrasonic vibration network; however, the average value is smaller. For the 


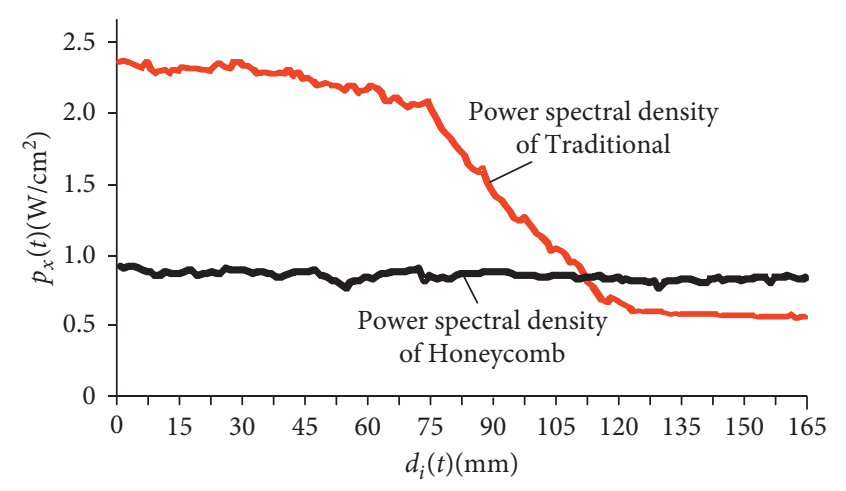

Figure 6: Power spectral density simulations of different vibration networks.

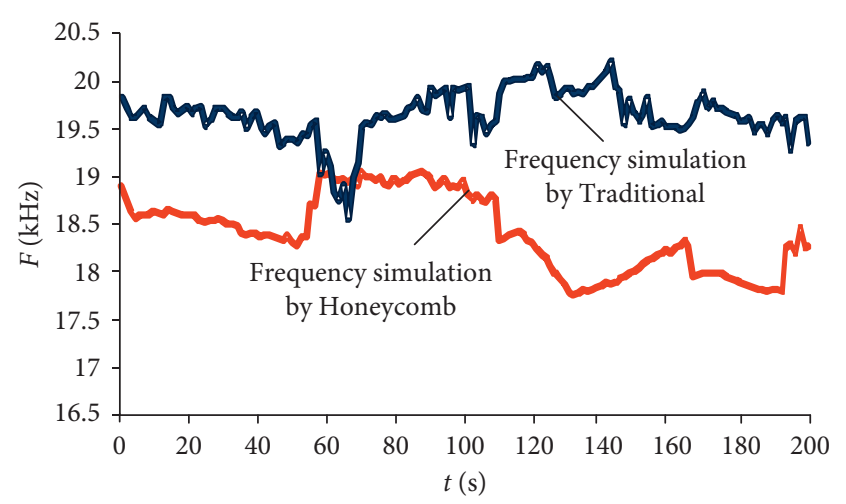

FIgURE 7: Frequency comparison of simulation in different ultrasonic vibration Networks.

resonant frequency of the traditional network, the grain size may be more uniform, and the number of holes in the aluminum alloy melt and the punching mode reduce.

\section{Experiments}

The effect of the honeycomb ultrasonic vibration network on the efficiency of ultrasonic-vibration-assisted casting system and casting quality was experimentally investigated, and $r_{f}(t)=f_{n}(t-1)-f_{n}(t-2)$ is the resonant frequency error.

\subsection{Experimental Environment}

4.1.1. Equipment and Materials for Honeycomb Ultrasonic Vibration-Assisted Casting. The experimental setup consists of two fourth generation programmable MCU ultrasonic vibration power supply 4 output in 1 , seven PTZ piezoelectric ceramic transducers, seven titanium alloy horns, two temperature control recorders, cylindrical crucible of $\varnothing 630 \mathrm{~mm}$ with $600 \mathrm{~mm}$ high, adjustable displacement device for ultrasonic horn, K-type thermocouple, Buehler grinder, AD-AM data acquisition module, Leica desktop metallographic microscope of Leica DM 6M and DM 8M model, 20 Aluminum alloy blocks of 2219 aluminum alloy blocks with $26 \mathrm{~kg}$ each, 10 kilograms Keller's corrosive agent, Al-Ti-B refiner with 10 kilograms, $\varnothing 630 \mathrm{~mm}$ iron crucible furnace for $\emptyset 630 \mathrm{~mm}$, and the other components. In summary, the experimental setup of ultrasonic vibration network-assisted casting with honeycomb is shown in Figure 8.

\subsubsection{Equipment and Materials for Traditional Ultrasonic} Vibration Network-Assisted Casting. The experimental setup consists of one fourth generation programmable MCU ultrasonic vibration power supply 4 output in 1 , four PTZ piezoelectric ceramic transducers, four titanium alloy horns, two temperature control recorders, cylindrical crucible of $\varnothing 630 \mathrm{~mm}$ with $600 \mathrm{~mm}$ high, adjustable displacement device for ultrasonic horn, K-type thermocouple, Buehler grinder, AD-AM data acquisition module, Leica desktop metallographic microscope of Leica DM6 M and DMi8 model, ten Aluminum alloy blocks of 2219 aluminum alloy blocks with $26 \mathrm{~kg}$ each, 10 kilograms Keller's corrosive agent, Al-Ti-B refiner with 10 kilograms, $\varnothing 630 \mathrm{~mm}$ iron crucible furnace for $\varnothing 630 \mathrm{~mm}$, and the other components. In summary, the experimental condition and setup of traditional ultrasonic vibration networkassisted casting is shown in Figures 9 and 10 .

4.1.3. Experimental Scheme. The ultrasonic-assisted casting experiment was carried out in the new ultrasonic vibration network environment. The output power, resonance frequency, temperature of the aluminum alloy melt, and penetration depth of the horn into the melt of the ultrasonic vibration power supply were obtained. The grain size was obtained by metallographic slicing. The specific experimental steps are as follows:

Step 1: according to Figure 8, a 7-horn ultrasonic vibration network environment with two ultrasonic vibration power supply system, 7 outputs, and diameter of $630 \mathrm{~mm}$ was developed to prepare for the experiment.

Step 2: inject aluminum alloy melt, heat it, and keep it between 700 and $735^{\circ} \mathrm{C}$. Data were acquired at intervals of $1 \mathrm{~s}$; the data acquisition cycle is $360 \mathrm{~s}, 20$ cycles, a total of 2 hour of ultrasonic-assisted casting experiment, acquisition of data information.

Step 3: repeat the steps mentioned in Step 2 for ordinary, intelligent, and honeycomb-based ultrasonic vibration networks, respectively.

$$
f_{N_{\mathrm{nod}}}(t)=\frac{\sum_{i=1}^{N_{\mathrm{nod}}}\left[f_{i}(t)+f_{i}(t-1)\right]}{14}+r_{f}(t-1) .
$$

4.2. Efficiency Analysis. The efficiency analysis includes a coverage analysis of the resonance frequency and power load, analysis of output power load of difference ultrasonic vibration power supplies, and frequency spectrum efficiency analysis of different ultrasonic power supplies.

4.2.1. Coverage Analysis. The amplitude does not vary in the traditional ultrasonic network designed by the coverage analysis. The position of the rod is adjusted. The distribution of the tool heads of aluminum alloy ingot with diameter $630 \mathrm{~mm}$ ultrasonic-assisted casting is shown in Figure 11. 


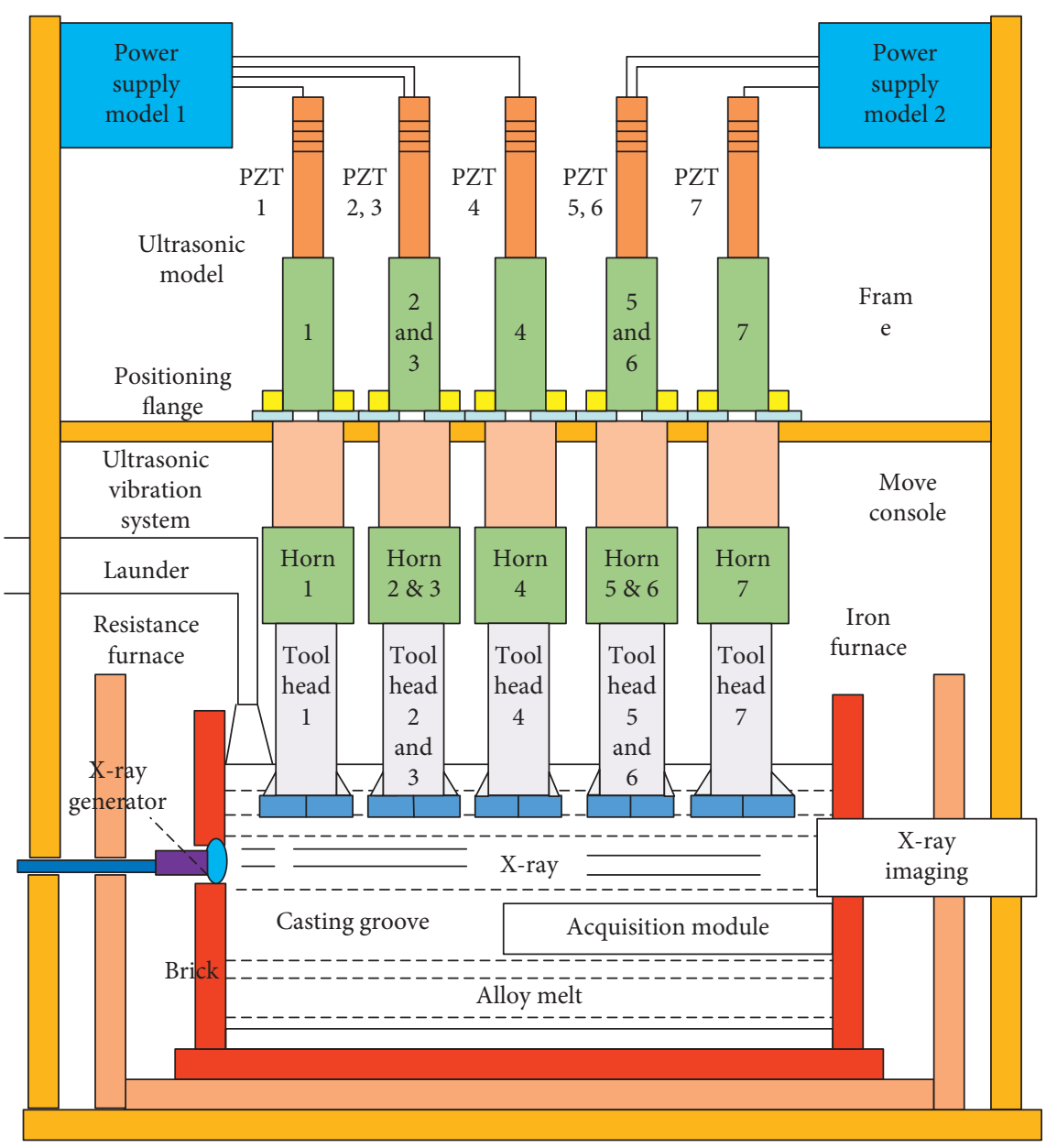

Figure 8: Experimental device of Honeycomb ultrasonic-assisted casting.

The minimum and maximum distances between the units are $199.8 \mathrm{~mm}$ and $282.33 \mathrm{~mm}$, respectively. The layers, number, and size of the ultrasonic vibration network unit of honeycomb structure are determined according to the size of the aluminum alloy ingot. The ultrasonic vibration network of seven nodes is constructed in the casting process of aluminum alloy with diameter $630 \mathrm{~mm}$. The distribution of the tool head is shown in Figure 12.

The distance between the nodes is fixed at $181.1 \mathrm{~mm}$; the theoretical distance is $179.97 \mathrm{~mm}$. The distance between the edges of the vibration units is $1.019 \mathrm{~mm}$. Although there is a distance between the tool heads, the vibration network of the two cellular units is connected by the ultrasonic action of edges; however, there is no connection between the cellular units.

The path loss of the vibration network is analyzed from two aspects of isotropy and anisotropy. The threshold is $2.94 \mathrm{db}$. Power ultrasound can affect the local temperature and pressure of the aluminum alloy melt in the process of ultrasonic-vibration-assisted casting. The area of 15 Celsius higher than the average melt temperature is the area covered by the vibration network. It is more convenient to obtain the temperature than the frequency during ultrasonic-vibrationassisted casting. The melt temperature can be detected along the path between the adjacent horns $i$ and $j$. To judge the coverage rate of vibration network, the coverage probability of horn $i$ in the honeycomb network decreases with an increase in the distance between the detection point and the center of vibration source. The distance between the middle point of self-adjacent node path and horn $j$ decreases, and the coverage rate of vibration network increases with the average coverage rate of 0.811 . The coverage efficiency of the traditional structure vibration network increases with an increase in the distance between the detection point and the center of vibration. When the distance is $90 \mathrm{~mm}$, the coverage rate is 0.2 ; when the distance is $150 \mathrm{~mm}$, it is 0.022 . Because the traditional ultrasonic vibration network does not improve the tool head, the shortest distance between the adjacent vibration units is $199.8 \mathrm{~mm}$, and there is a blind area, which results in the average coverage rate of 0.476 . The experimental results in Figure 13 show that the coverage probability of the traditional vibration network is much lower than that of the new ultrasonic vibration network.

4.2.2. Analysis of Power Ultrasonic Action. Using the X-ray acquisition module shown in Figure 14, the X-ray image of flat thin cylindrical fluid, $2.49 \mathrm{~cm}-7.61 \mathrm{~cm}$ below the plane of multiple tool heads, was obtained during the ultrasonicvibration-assisted casting. The results of Figure 14 show that the temperature distribution of the honeycomb-structured 


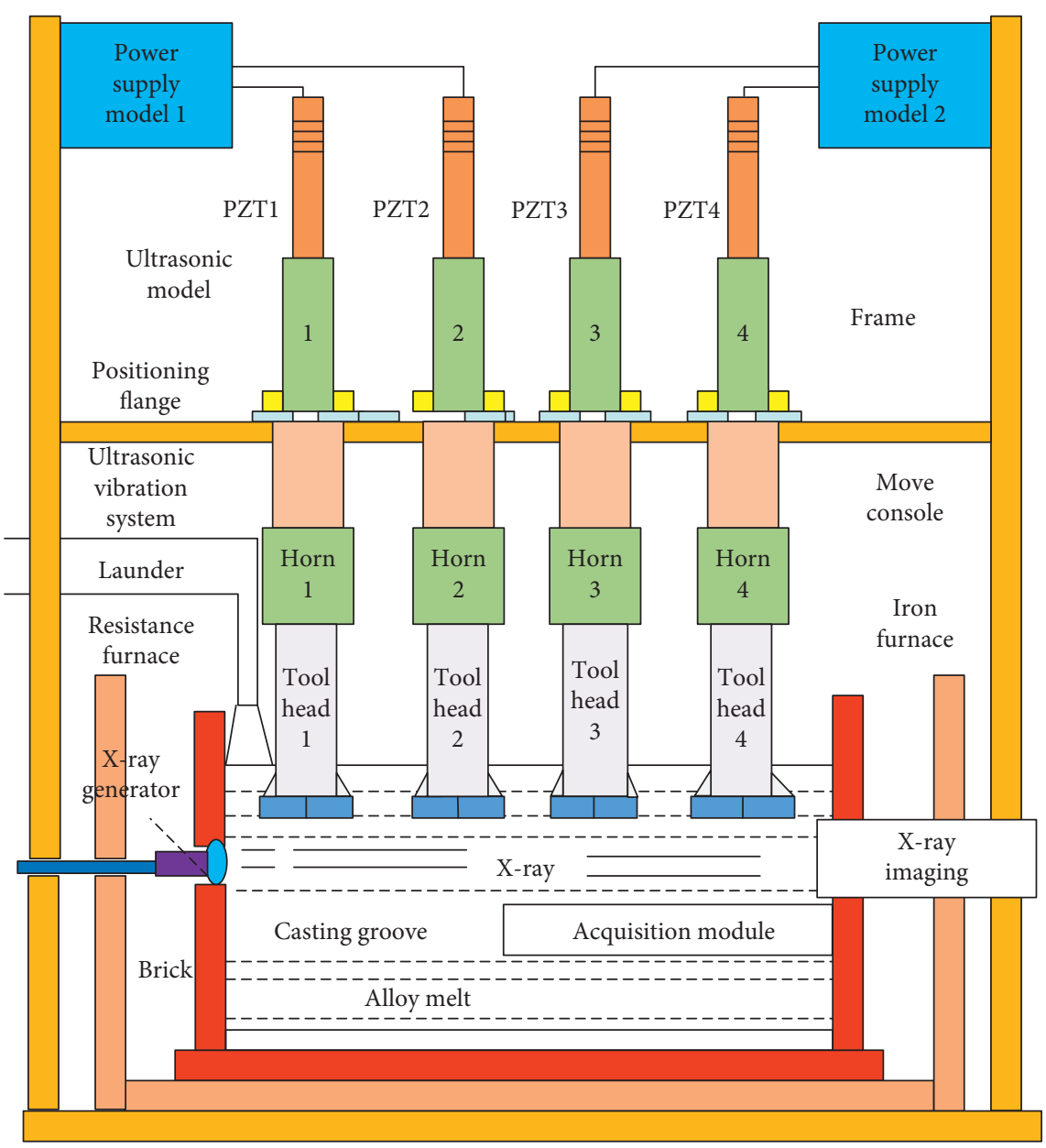

FIGURE 9: Experimental installation of ultrasonic vibration network with traditional.

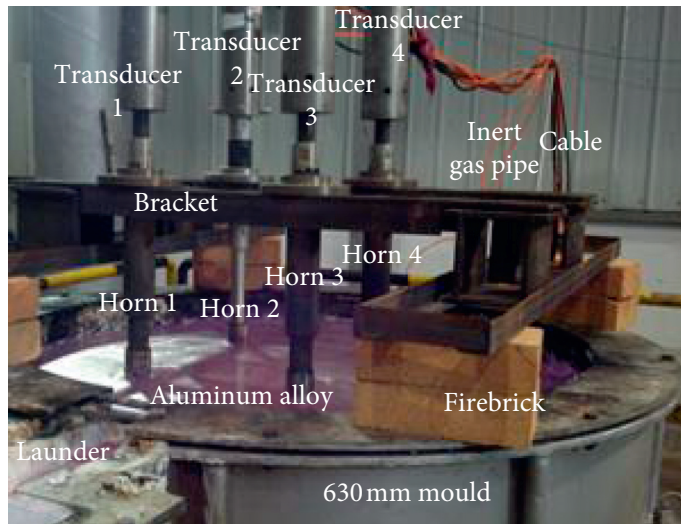

FIgURE 10: Experimental device of traditional ultrasonic-assisted casting.

ultrasonic vibration network is uniform, and the efficiency is higher than that of the traditional ultrasonic vibration network.

4.2.3. Frequency Spectrum Efficiency Analysis. The number of vibration sources per unit area is $N_{\text {unit }}=\lambda_{B}\left(\mathrm{n} / \mathrm{cm}^{2}\right)$, and the frequency spectrum efficiency per unit area is obtained.

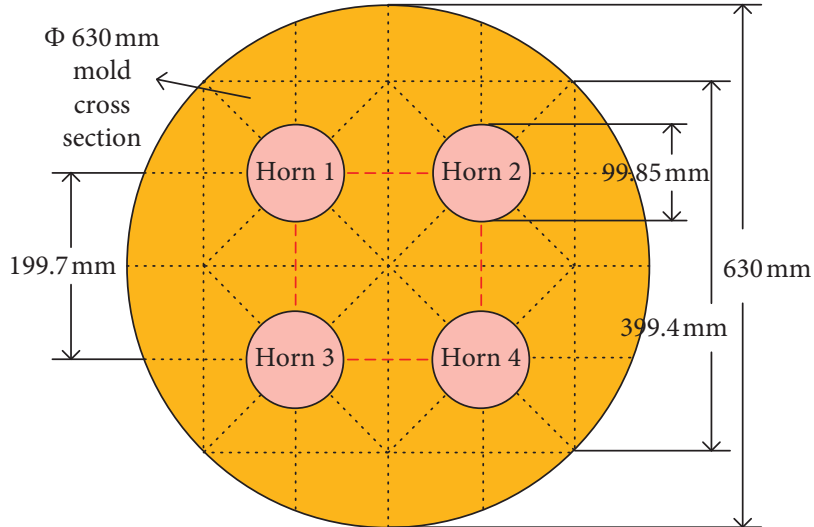

FIGURE 11: Distribution of traditional four-unit ultrasonic vibration network.

$$
\begin{aligned}
\Omega\left(\lambda_{u}, v_{i}, \varepsilon_{i}, \tau_{i}, \theta_{i}\right)= & \lambda_{B} \cdot \Lambda\left(\lambda_{u}, v_{i}, \varepsilon_{i}, \tau_{i}, \theta_{i}\right), \\
\Omega(\lambda u, \nu, \varepsilon, \tau, \theta)= & \frac{1}{2} \int_{1}^{590} \int_{591}^{735} \frac{(\beta-2)^{\tau-1} e^{-(\beta-2) / \theta}\left[1-(1+z)^{-4}\right] \varepsilon \rho \lambda_{B}}{\Gamma(\tau) \theta^{\tau}\left(\nu^{-\varepsilon}-\psi^{-\varepsilon}\right) z \Theta \lambda_{B}} \\
& \cdot\left\{1+\left(\pi \Theta \lambda_{B}\right)^{1 / 42}\left[\Gamma\left[\frac{\varepsilon \pi \theta}{2} \Theta \lambda, \psi^{2}\right]\right]\right\} \mathrm{d} z \mathrm{~d} \beta,
\end{aligned}
$$




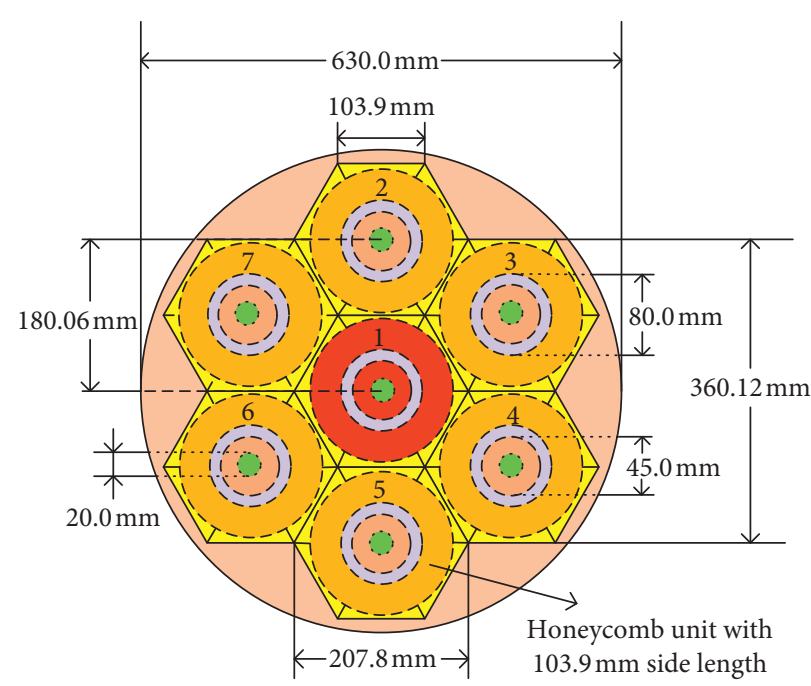

FIGURE 12: Distribution of two layers with seven-node honeycomb-structured ultrasound vibration network.

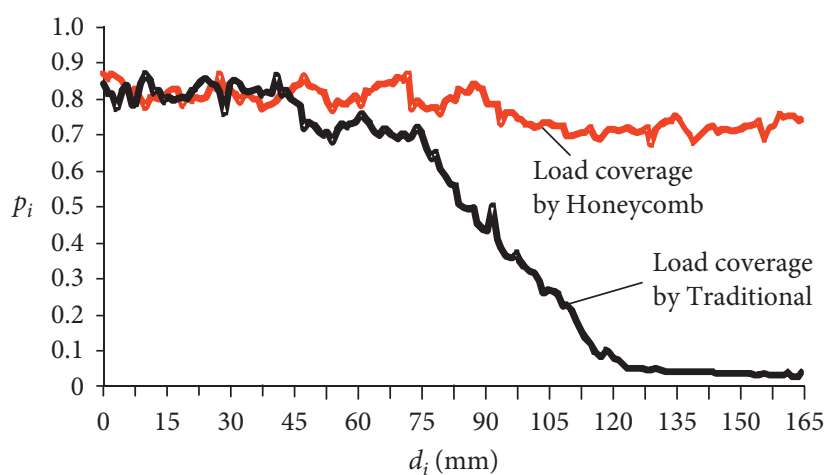

FIGURE 13: Experiments of load coverage probability of a new ultrasonic vibration network unit.

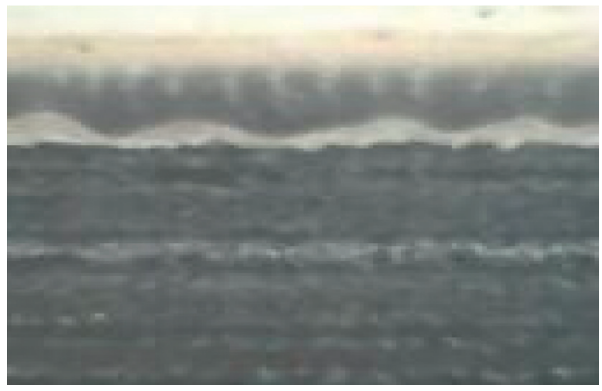

(a)

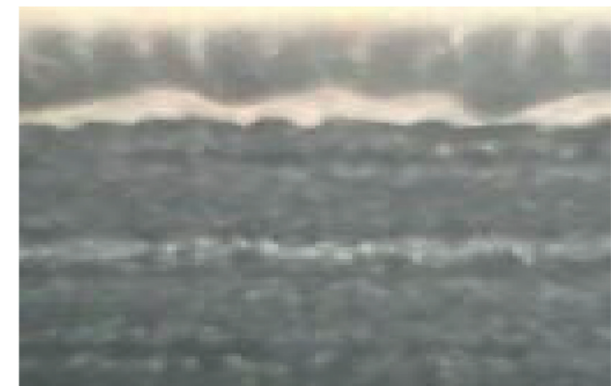

(b)

Figure 14: X-ray image of molten aluminum alloy. (a) Honeycomb network. (b) Traditional network.

where

$$
\begin{aligned}
\Theta & =-1+(1+z)^{-8}+6 z^{1 / 3} B_{0}^{b}\left(\frac{1}{6}, 2, \frac{5}{3}\right), \\
B_{0}^{b}\left(\frac{1}{6}, 2, \frac{5}{3}\right) & =\int_{0}^{b-10 / 3}(1-t)^{23 / 3} \mathrm{~d} t, \\
\Gamma(-y, x) & =\int_{x}^{\infty} \frac{e^{-t}-\sum_{i=0}^{N_{\text {nod }}}(-1)^{i} t^{i} i i !}{t^{y+1}} \mathrm{~d} t .
\end{aligned}
$$

The frequency spectrum efficiency of the seven-bar honeycomb structure with a diameter of $630 \mathrm{~mm}$ is shown in Figure 15.

The experimental results show that the area frequency spectrum efficiency of the new vibration network is closely related to the unit fractal. The frequency spectrum efficiency of the cellular network is $(54.61 \%, 78.49 \%)$ and stable. The frequency spectrum efficiency of the traditional structural network is $(3.74 \%, 94.74 \%)$. The efficiency decreases sharply when the distance from the center of horn is greater than 


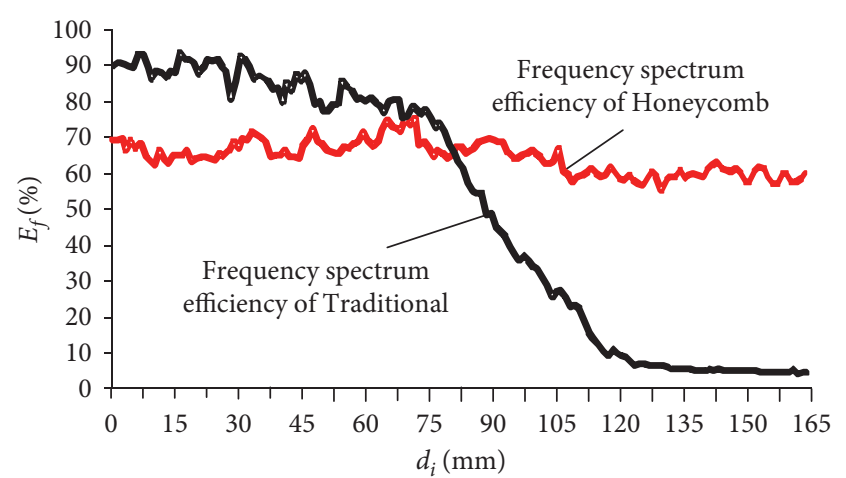

Figure 15: Frequency spectrum efficiency comparison of different vibration networks.

$75 \mathrm{~mm}$. The frequency spectrum efficiency of cellular network is $3.74 \%$ when the distance is increase to $120 \mathrm{~mm}$, and the efficiency of the frequency spectrum is higher than that of the traditional vibration network.

4.3. Casting Quality. In summary, the experimental condition and setup of ultrasonic vibration network-assisted casting with the mould were carried out at $735^{\circ} \mathrm{C}$ using the traditional network and the honeycomb-structured vibration network. As shown in Figure 16, eight cuts were made along $\mathrm{C} 1$ to $\mathrm{C} 8$. Aluminum alloy slices with a volume of $1 \mathrm{~cm}^{3}$ were obtained and numbered from $\mathrm{B} 1$ to $\mathrm{B} 4$. The samples were polished, cleaned by alcohol, corroded by Keller reagent, and dried in air. The metallographic and surface imaging of the samples is compared in Figures 17 and 18.

The area method is to obtain the number of grains in a given area through the Lycra metallographic analysis microscope and divide the designated area by the number of grains to obtain the average size of the aluminum alloy melt grains. The accuracy is $0.0025 \mu \mathrm{m}$, and the reproducibility of the measurement results is less than $0.005 \mu \mathrm{m}$. The cross-section method is to calculate the cross-section points of the known length test line (or grid) and the grain interface to determine the grain size. Its accuracy is also $0.0025 \mu \mathrm{m}$. The accuracy of the crosssection method is a function of the calculated crosssection points or intercept. Through effective statistical results, the accuracy of $\pm 0.25 \mu \mathrm{m}$ can be achieved. The error of minimum reproducibility value and maximum reproducibility value is less than $0.005 \mu \mathrm{m}$. For the same accuracy level because it does not need to accurately measure the number of cross-section points or interceptions, the cross-section method is faster than the area method. In this paper, firstly, the grain size is measured by area division, and then the grain size is measured by the cross-section method, and the average value of the two is taken as the grain size. So, the grain sizes of 2219 aluminum alloy under different ultrasonic-vibration-network environments are shown in Table 3.

The grain size is uniform, and the casting quality is improved under the honeycomb vibration network

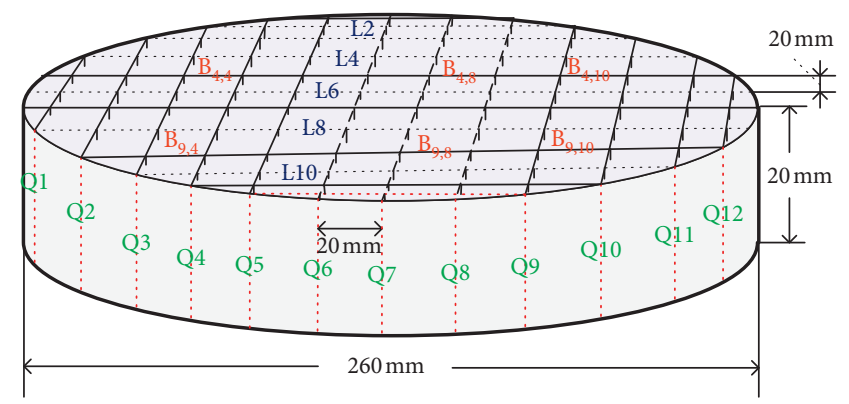

FIgURE 16: Sampling slice acquisition.

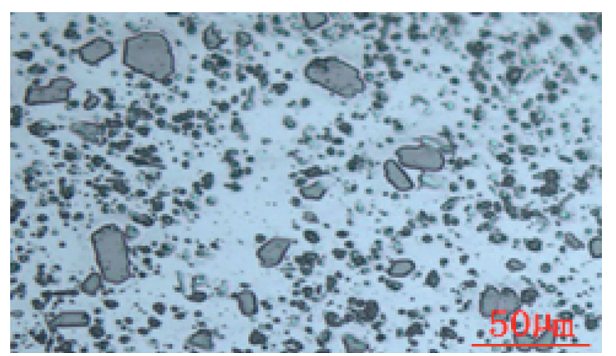

FIGURE 17: Metallography of traditional network.

TABle 3: Effects of different networks on grain size.

\begin{tabular}{lcc}
\hline Position & \multicolumn{2}{c}{ Grain size with different vibration } \\
& networks $(\mu \mathrm{m})$ \\
\hline B1 & Traditional & Honeycomb \\
B2 & 174.23 & 145.91 \\
B3 & 176.01 & 136.26 \\
B4 & 173.86 & 143.45 \\
Average & 199.06 & 156.52 \\
B5 & 180.79 & 145.54 \\
B6 & 186.51 & 150.74 \\
B7 & 177.32 & 147.81 \\
B8 & 178.86 & 139.78 \\
B9 & 175.83 & 134.74 \\
average & 173.26 & 145.99 \\
\hline
\end{tabular}

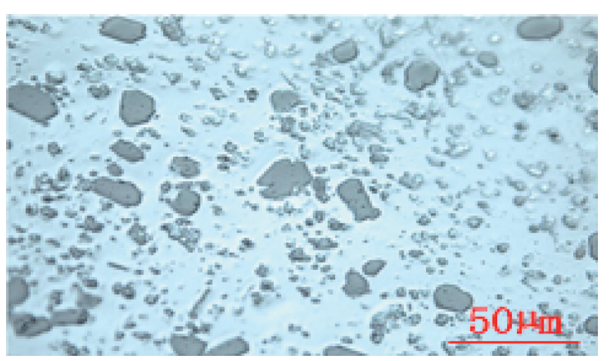

FigURE 18: Metallography of honeycomb network.

environment. Table 3 and Figure 16 show that the ultrasonic vibration network of the honeycomb structure is superior to that of the traditional vibration network. 


\section{Conclusions}

(1) The frequency spectrum efficiency of the honeycomb-structured network is higher than that of the traditional network. The frequency spectrum efficiency range of cellular network is $54.61-78.49 \%$. The frequency spectrum efficiency of different detection points varies slightly and steadily. The frequency spectrum efficiency of the traditional network is $3.741-94.75 \%$. With an increase in the distance between the test point and the vibration center, the efficiency of output power decreases rapidly, and the frequency efficiency of the vibration network decreases rapidly.

(2) The honeycomb reference efficiency increases with an increase in the number of layers of the vibration network. When there are two layers, the spatial efficiency is $64.02 \%$; when there are eight layers, the spatial efficiency is $78.14 \%$. The edge length of the vibration network unit module changes with the change in the number of layers of network node and ingot size. The volume of the vibration network changes from $9820.45 \mathrm{~cm}^{3}$ to $12089.05 \mathrm{~cm}^{3}$, and the volume efficiency increases gradually.

(3) The power spectral density of the honeycombstructured ultrasonic network ranges from 0.736 to $0.902 \mathrm{~W} / \mathrm{cm}^{2}$, and the power spectral density of traditional ultrasonic network ranges from 0.07 to $2.35 \mathrm{~W} / \mathrm{cm}^{2}$. The power spectral density of honeycomb-structured ultrasonic network is higher than that of the traditional ultrasonic network.

(4) The nucleation rate of 2219 aluminum alloy honeycomb structure increased during the ultrasonicvibration-assisted casting. The average grain size was $145.939 \mathrm{~mm}$, while the average grain size of the traditional vibration network assisted casting was $180.79 \mathrm{~m}$, which reduced by 34.88 microns, and the quality of assisted casting was improved.

The new ultrasonic vibration network shape still has several limitations. In the normal casting temperature range, the output power and frequency of power supply fluctuate randomly with a decrease in the melt temperature. The problem of power and frequency stability control of the ultrasonic vibration network should be studied in the future. In addition, there is no optimal structure for the ultrasonic vibration network. A better structure of ultrasonic vibration network for foundry needs to be improved and optimized continuously.

\section{Data Availability}

The data supporting the results of this study are named mat.4913286.v6, which are stored on the BAIDU disk: https://pan.baidu.com/s/1EmR3RrxhjwvWpTHxmLQP_g password: 8gyt. The research results are included in the supplementary information files, including 3.1 Efficiency Analysis, 3.1.1 coverage Analysis, 3.1.2 Analysis of Power Ultrasonic Action, 3.1.3 Frequency Spectrum Efficiency
Analysis, and 3.2 Casting Quality and Conclusions and the contents of simulation results, the experimental results, and images and tables are included in the file name of "OKmat.4913286.v6.20200908.docx". If the researchers or reader could not get the results and data of this manuscript, they can contact the author via e-mail: chenxiwen001@qq.com. All data used to support this study can be used and are not prohibited. The data results of the paper are only used for reference in scientific research and engineering applications. Within 6 to 12 months after publication of this paper, data can be obtained from the corresponding author upon request via e-mail: chenxiwen001@qq.com .

\section{Conflicts of Interest}

The authors declare that there are no conflicts of interest regarding the publication of this paper.

\section{Acknowledgments}

This work was partially supported by the National Basic Research Development Program of China (Grant no. 2012CB619504), the National Natural Science Foundation of China (Grant nos. U1637601 and 51575539), the Hunan Province Overseas Research and Training Program for University Prominent, the Excellent Scientific and Technological Innovation Team of Central South University, and the authors also thank academician Professor Gui Weihua of the Chinese Academy of Engineering for the helpful comments.

\section{Supplementary Materials}

(1) The simulation result information mainly covers the simulation results of different vibration network output powers and resonance frequencies. The simulation period is 1800 seconds, and there are a total of 1440 simulation results. (2) The experimental results of different ultrasonic vibration networks mainly include the experimental results of the power supply and resonant frequency of the power supply in different vibration networks. The experimental data acquisition period is also 1800 seconds, and there are a total of 1440 experimental results. (3) Experimental equipment parameter information, including basic parameters of different power sources in two different ultrasonic vibration networks. (4) The contents of simulation results and experimental results are included in 4913286.v1.docx file. (Supplementary Materials)

\section{References}

[1] L. Zhang and B. Zhu, "Numerical simulation for 7050 aluminum alloy cast with ultrasonic treatment," Materials for Mechanical Engineering, vol. 37, no. 1, pp. 85-92, 2013.

[2] X. Li, K. Li, M. Chen, and S. Zhao, "Effect of ultrasonic vibration on cooling time and solidification structure of 7050 aluminum alloy melt," Materials Science and Engineering of Powder Metallurgy, vol. 16, no. 2, pp. 249-254, 2011. 
[3] Y. Cui and X. Li, "Effects of ultrasonic on micro-structures and solid solubility of main solute elements in aluminum alloy 7050," Journal of Central South University (Science and Technology), vol. 43, no. 9, pp. 3420-3425, 2012.

[4] Q. Chen, W. Li, T. Wu, and J. Wu, "Influence of numerical integration on error of ultrasonic flow meter," Journal of Xian Jiaotong University, vol. 46, no. 9, pp. 21-25, 2012.

[5] L. Yan, Z. Yang, F. Gao, and J. Liu, "Power spectral density compensation algorithm for signal clipping in vibration test," Journal of Vibration, Measurement \& Diagnosis, vol. 35, no. 4, pp. 690-696, 2015.

[6] J. Zhong, L. Qi, M. Li, Z. Zhao, and H. Li, "Automatic classification of pyrocarbon texture under polarized light microscope based on artificial neural network," Journal of Xi'an Jiao University, vol. 44, no. 7, pp. 46-49, 2010.

[7] H. M. Vishwanatha, J. Eravelly, C. S. Kumar, and S. Ghosh, "Microstructure and mechanical properties of aluminumalumina bulk nanocomposite produced by a novel two-step ultrasonic casting technique," Metallurgical and Materials Transactions A, vol. 47, no. 11, pp. 5630-5640, 2016.

[8] F. Du, J. Hong, and L. Baotong, "Contact parameter estimation with ultrasonic method," Journal of Xi'an Jiao University, vol. 47, no. 3, pp. 19-23, 2013.

[9] A. Stcinwolf, "Shaker random testing with low kurtosis: review of methods and application for sigma limiting," Shock and Vibration, vol. 17, pp. 219-231, 2010.

[10] S. Wu, G. Zhong, L. Wan, P. An, and Y. Mao, "Microstructure and properties of rheo-diecast $\mathrm{Al}-20 \mathrm{Si}-2 \mathrm{Cu}-1 \mathrm{Ni}-0.4 \mathrm{Mg}$ alloy with direct ultrasonic vibration process," Transactions of Nonferrous Metals Society of China, vol. 20, no. S3, pp. 763$767,2010$.

[11] T. Wen, L. Wei, X. Chen, and C.-L. Pei, "Effects of ultrasonic vibration on plastic deformation of AZ31 during the tensile process," International Journal of Minerals, Metallurgy, and Materials, vol. 18, no. 1, pp. 70-76, 2011.

[12] A. Nestler and A. Schubert, "Surface properties in ultrasonic vibration assisted turning of particle reinforced aluminum matrix composites," Procedia CIRP, vol. 13, pp. 125-130, 2014.

[13] J.-P. Lai, R.-P. Jiang, H.-S. Liu, X.-L. Dun, Y.-F. Li, and X.-Q. Li, "Influence of cerium on microstructures and mechanical properties of $\mathrm{Al}-\mathrm{Zn}-\mathrm{Mg}-\mathrm{Cu}$ alloys," Journal of Central South University, vol. 19, no. 4, pp. 869-874, 2012.

[14] L.-H. Zhang, J. Yu, and X.-M. Zhang, "Effect of ultrasonic power and casting speed on solidification structure of 7050 aluminum alloy ingot in ultrasonic field," Journal of Central South University of Technology, vol. 17, no. 3, pp. 431-436, 2010.

[15] X. W. Chen, X. Q. Li, and S. H. Shen, "The design and application of new control model for ultrasonic vibration power supply," Journal of Vibration, Measurement \& Diagnosis, vol. 37, no. 4, pp. 703-708, 2017.

[16] J. Xie, X. Q. Li, R. Q. Li, and R. P. Jiang, "Homogenization process and the second phases evolution of 2219 aluminum alloy treated by ultrasonic," Journal of Central South University (Science and Technology), vol. 48, no. 9, pp. 2288-2293, 2017.

[17] Y. Y. He, L. Rui, H. Y. Li, S. Wang, and X. Lu, "Short-term power load probability density forecasting method using kernel-based support vector quantile regression and Copula theory," Applied Energy, vol. 185, no. 1, pp. 254-266, 2017.

[18] H. Jiang, A. S. E. Chong, Y. Ueda, and M. Wiercigroch, "Grazing-induced bifurcations in impact oscillators with elastic and rigid constraints," International Journal of Mechanical Sciences, vol. 127, no. 9, pp. 204-214, 2017.
[19] T. R. Chen, Y. L. Lee, H. T. Liu, S. M. Chen, and H. Z. Chang, "Study of contactless power supply for spindle ultrasonic vibrator," IOP Conference Series: Earth and Environmental Science, vol. 94, no. 1, pp. 2099-2103, 2017.

[20] X. W. Chen, X. Q. Li, and R. Q. Li, "Ultrasonic power load forecasting based on BP neural network," Journal of The Institution of Engineers (India): Series C, vol. 101, no. 23, pp. 383-390, 2020. 